\title{
MOMENT MEASURES OF MIXED EMPIRICAL RANDOM POINT PROCESSES AND MARKED POINT PROCESSES IN COMPACT METRIC SPACES. I
}

UDC 519.21

\author{
M. G. SEMEǏKO
}

\begin{abstract}
Moment measures of mixed empirical random point processes and marked point processes are investigated by using probability generating functions of random counting measures.
\end{abstract}

\section{INTRODUCTION}

An interest in the theory of and statistical inferences for random point processes and marked point processes has grown essentially over last decade because of the applications found in spherical stochastic geometry [1], stereology [2], mathematical morphology [3] as well as biology, ecology, cosmology, and economics.

In contrast, mixed empirical processes generated by independent identically distributed elements (random variables) $x_{1}, x_{2}, \ldots, x_{N}$ assuming values in a measurable space $\left(X, \mathfrak{A}_{X}\right)$ occupy rather little space in the theory of point processes. The key assumption of the theory of mixed empirical point processes is that the number of elements $N$ is a nonnegative random variable assuming only integer values and that $N$ is independent of the random elements $x_{1}, x_{2}, \ldots, x_{N}$. If the number of elements $N$ is a nonrandom fixed number, say $N=n$, then the independent and identically distributed random elements $x_{1}, x_{2}, \ldots, x_{n}$ generate an empirical ( $n$-sampled) point processes in the measurable space $\left(X, \mathfrak{A}_{X}\right)$ (see [4]). The notion of an empirical point process and mixed empirical point process was introduced by Karr 44. Empirical processes have been studied by Karr [4, Csörqö and Révész [5], Gaenssler [6], Gaenssler and Stute [7, Pollard 8, and Serfling [9].

The theory of mixed empirical marked point processes is more general and allows one to study various problems of stochastic geometry, especially if the geometric structures are not uniformly distributed in the spaces $\mathbf{R}^{2}, \mathbf{R}^{3}$, or $S^{2}$.

In this paper, we continue an investigation of properties of mixed empirical ordered point processes and ordered marked point processes initiated in [10]-[12]. The main focus of attention is the properties of moment measures of mixed empirical ordered point processes and those of ordered marked point processes in compact metric spaces constructed from samples obtained by simple random choice from a population without repetition.

The paper is organized as follows. The first part consists of sections 26 and deals with mixed empirical random ordered point processes in compact metric spaces. Section 2 contains main definitions and results of the theory of random point processes and marked point processes [10, 13]. Section 3 provides main notions of the theory of mixed empirical random ordered point processes.

2010 Mathematics Subject Classification. Primary 60G55.

Key words and phrases. Mixed empirical point processes, marked point processes, probability generating functions, moment measures. 
Joint and marginal probability generating functions of random empirical counting measures of ordered point processes are constructed in Section 4. Moment generating functions are widely used tools for studying moment measures, mixed moment measures, and factorial moment measures of ordered point processes.

Models of mixed empirical Poisson and negative binomial random ordered point processes are constructed in Sections 5 and 6. Various moment measures of these ordered point processes are presented in Sections 5 and 6 .

The second part of the paper consists of Sections 7-10 and deals with mixed empirical random ordered marked point processes in compact metric spaces. The main notions of the theory of mixed empirical random ordered marked point processes with independent marks in compact metric spaces are considered in Section 7.

Moment measures of a mixed empirical random ordered marked point processes are discussed in Section 8.

The models of mixed empirical Poisson and negative binomial random ordered marked point processes are constructed in Sections 9 and 10. Various moment measures are studied for these point processes.

The list of references is identical for both parts.

This publication is devoted to the memory of Professor Yu. I. Petunin, who was the scientific advisor for my Ph.D. dissertation. When writing this paper, I used his valuable comments and remarks.

\section{MAin NOTIONS OF THE THEORY OF RANDOM POINT PROCESSES AND MARKED POINT PROCESSES}

Below we list the main notions of the theory of point processes and marked point processes to be used throughout the paper; also see [10, 13.

Let $\left(X, \mathfrak{A}_{X}\right)$ be a measurable space and let the $\sigma$-algebra $\mathfrak{A}_{X}$ contain all singleton subsets $\{x\}$ of the space $X$. We say that a measurable space $\left(X, \mathfrak{A}_{X}\right)$ is endowed with a structure $\mathfrak{B}_{X}$ of bounded sets if the following conditions hold [14]:

(1) $\mathfrak{B}_{X}$ is a hereditary class;

(2) $\mathfrak{B}_{X}$ is closed under finite unions of its elements;

(3) $\mathfrak{B}_{X}$ is a covering of the space $X$;

(4) The class of bounded measurable sets $\mathfrak{E}_{X}=\mathfrak{A}_{X} \cap \mathfrak{B}_{X}$ is cofinal in $\mathfrak{B}_{X}$ under inclusions; that is, if $B_{X} \in \mathfrak{B}_{X}$, then there exists $F_{X} \in \mathfrak{E}_{X}$ such that $B_{X} \subset F_{X}$.

Definition 2.1. A measurable class $\left(X, \mathfrak{A}_{X}\right)$ endowed with a structure of bounded sets $\mathfrak{B}_{X}$ is called a bounded space and denoted by $\left(X, \mathfrak{A}_{X}, \mathfrak{B}_{X}\right)$ [14].

Definition 2.2. A multiset

$$
E=\left(x_{1}, x_{2}, \ldots, x_{n}, \ldots\right)
$$

of a bounded space $\left(X, \mathfrak{A}_{X}, \mathfrak{B}_{X}\right)$ (that is, some points of the space $X$ may appear in $E$ several times) is called a thinned set if the intersection with an arbitrary measurable bounded set $B_{X} \in \mathfrak{E}_{X}$ contains only a finite number of elements, that is

$$
N\left(E, B_{X}\right)=\operatorname{card}\left[E \cap B_{X}\right]<\infty,
$$

where card $\left[E \cap B_{X}\right]$ is the number of elements in the set $E \cap B_{X}$.

Let $N\left(E, B_{X}\right)$ be a counting measure in the class of measurable bounded subsets $B_{X}$ of the space $X$ ( $N$ assumes only nonnegative positive values, that is, $N\left(E, B_{X}\right) \in Z_{+}$). We say that a point $x$ of a thinned set $E$ is an atom of the measure $N\left(E, B_{X}\right)$ if

$$
N(E,\{x\})>0 \text {. }
$$


For an arbitrary atom $x$ of a measure $N$, the value $N(E,\{x\})$ equals the multiplicity of the point $x$.

Thus a counting measure $N\left(E, B_{X}\right)$ and a thinned set $E$ are such that

$$
\begin{gathered}
N\left(E, B_{X}\right)=\sum_{x \in E} N(E,\{x\}) I_{B_{X}}(x), \\
E=\{x: x \in X, 0<N(E,\{x\})<\infty\}
\end{gathered}
$$

(see [15]), where $I_{B_{X}}(x)$ denotes the characteristic function of the set $B_{X}$. A thinned set $E$ can be viewed as a subset of the space $X \times\{1,2,3, \ldots\}$, where the second component (index) means the multiplicity of a point of the space $X$ [14].

Definition 2.3. A thinned set $E$ of a bounded space $\left(X, \mathfrak{A}_{X}, \mathfrak{B}_{X}\right)$ is called simple if $E=\{x: x \in X, N(E,\{x\})=1\}$. In particular, a simple thinned set does not contain multiple points.

Definition 2.4. A counting measure $N\left(E, B_{X}\right)$ is called simple if it admits the following representation

$$
N\left(E, B_{X}\right)=\sum_{x \in E} I_{B_{X}}(x)
$$

It is clear that a simple thinned set $E$ corresponds to a simple counting measure $N\left(E, B_{X}\right)$.

Definition 2.5. A counting measure $N\left(E, B_{X}\right)$ is called locally finite (or a Radon measure) in a bounded space $\left(X, \mathfrak{A}_{X}, \mathfrak{B}_{X}\right)$ if $N\left(E, B_{X}\right)<\infty$ for all $B_{X} \in \mathfrak{E}_{X}$ [16].

Denote by $\mathcal{E}$ the class of all thinned subsets $E$ of a bounded space $\left(X, \mathfrak{A}_{X}, \mathfrak{B}_{X}\right)$. Let $B_{X}$ be an arbitrary measurable bounded set $\left(B_{X} \in \mathfrak{E}_{X}\right)$. A locally finite measure $N\left(E, B_{X}\right)$ is a mapping defined in the class $\mathcal{E}$ of all thinned set $E$ if

$$
N_{B_{X}}(\cdot)=N\left(\cdot, B_{X}\right): \mathcal{E} \longrightarrow Z_{+} \cdot
$$

Considering the class $\mathcal{E}$ of all thinned sets $E$ as a new main space, denote by $\mathfrak{X}$ the minimal $\sigma$-algebra of subsets of the space $\mathcal{E}$ formed by all subsets of the form

$$
\left\{E: N\left(E, B_{X}^{i}\right)=n_{i}, i=1,2, \ldots, m\right\},
$$

where $m \in \mathbb{N}, B_{X}^{i} \in \mathfrak{E}_{X}, n \in Z_{+}$. Thus $\mathfrak{X}$ is the minimal $\sigma$-algebra of subsets of the space $\mathcal{E}$ for which all mappings $N\left(E, B_{X}\right)$ are measurable with respect to $\mathfrak{X}$ for all $B_{X} \in \mathfrak{E}_{X}($ see [15]).

Definition 2.6. A probability space $(\mathcal{E}, \mathfrak{X}, \mathrm{P})$ is called a random point process in a bounded space $\left(X, \mathfrak{A}_{X}, \mathfrak{B}_{X}\right)$, where $\mathrm{P}$ is a probability measure defined in the $\sigma$-algebra $\mathfrak{X}$ [13. In this case, $X$ is called the space of states of a point process.

Definition 2.7. A random point process $(\mathcal{E}, \mathfrak{X}, \mathrm{P})$ in a bounded space

$$
\left(X, \mathfrak{A}_{X}, \mathfrak{B}_{X}\right)
$$

is called finite if

$$
\mathrm{P}\{E: N(E, X)<\infty\}=1 \text {. }
$$

Definition 2.8. A random point process $(\mathcal{E}, \mathfrak{X}, \mathrm{P})$ is called simple in a bounded space $\left(X, \mathfrak{A}_{X}, \mathfrak{B}_{X}\right)$ if every trajectory $E$ of the process is a simple thinned set in the space $X$.

Following [17, we say that a point process does not have multiple points with probability one if

$$
\mathrm{P}\{E: N(E,\{x\}) \leq 1, x \in X\}=1 .
$$


Definition $2.9([13)$. A random process $(\mathcal{E}, \mathfrak{X}, P)$ is called a simple ordered point process (ordered point process) in a bounded space $\left(X, \mathfrak{A}_{X}, \mathfrak{B}_{X}\right)$ if $X$ is a linear ordered space [18 and almost all its trajectories $E \in \mathcal{E}$ is a finite or countable simple thinned set (sequence) in the space $X$, that is $E=\left(x_{1}, \ldots, x_{n}\right)$ (respectively, $E=\left(x_{1}, \ldots, x_{n}, \ldots\right.$ ) or $\left.E=\left(\ldots, x_{-n}, \ldots, x_{-1}, x_{0}, x_{1}, \ldots, x_{n}, \ldots\right)\right)$ such that $x_{k}<x_{m}$ if $k<m$.

Definition $2.10([13])$. We say that a random process $(\mathcal{E}, \mathfrak{X}, \mathrm{P})$ is a simple unordered point process in a bounded space $\left(X, \mathfrak{A}_{X}, \mathfrak{B}_{X}\right)$ if almost every trajectory $E$ is an unordered simple thinned set in the space $X: E=\left\{x_{(1)}, \ldots, x_{(n)}\right\}$. (The subscripts are used for elements of the set $E$ to distinguish between the points and do not indicate any order for these points.)

Every random event is viewed in the theory of marked point processes as an ordered pair $[x ; k]$ formed by the state $x$ and its mark $k$. The point $x$ is an element of the space of states $X$ and indicates the place where the event may occur, while the mark $k$ is an element of the space of marks $K$ and corresponds to a certain numerical characteristic of the event (for example, its intensity, speed, or power). Thus the phase space $Y$ of such a marked point process is the Cartesian product of the spaces $X$ and $K: Y=$ $X \times K$, where $K$ is a semi-ordered (partially ordered) set 18 endowed with a $\sigma$-algebra of measurable sets $\mathfrak{A}_{K}$ and with a structure of bounded sets $\mathfrak{B}_{K}$.

As usual, the $\sigma$-algebra in the space $Y$ is the Cartesian product $\mathfrak{A}_{Y}=\mathfrak{A}_{X} \otimes \mathfrak{A}_{K}$ of the $\sigma$-algebras $\mathfrak{A}_{X}$ and $\mathfrak{A}_{K}$. In the measurable space $\left(Y, \mathfrak{A}_{Y}\right)$, we introduce a structure of bounded sets $\mathfrak{B}_{Y}=\mathfrak{B}_{X} \odot \mathfrak{B}_{K}$ for which a set $B_{Y} \subset Y$ is bounded if and only if there are bounded sets $B_{X} \in \mathfrak{B}_{X}$ and $B_{K} \in \mathfrak{B}_{K}$ such that $B_{Y} \subseteq B_{X} \times B_{K}$. All axioms of a structure of bounded sets are satisfied for $\mathfrak{B}_{Y}$ and thus $\left(Y, \mathfrak{A}_{Y}, \mathfrak{B}_{Y}\right)$ is a bounded space.

Definition 2.11. A random point process $\left(\mathcal{E}^{*}, \mathfrak{X}^{*}, P^{*}\right)$ in a bounded space $\left(Y, \mathfrak{A}_{Y}, \mathfrak{B}_{Y}\right)$ is called a marked point process; here $\mathcal{E}^{*}=\left\{E^{*}\right\}$ is the class of all thinned sets $E^{*}=$ $\left(\left[x_{1} ; k_{1}\right], \ldots,\left[x_{n} ; k_{n}\right], \ldots\right)$ of a bounded space $\left(Y, \mathfrak{A}_{Y}, \mathfrak{B}_{Y}\right)$.

Definition 2.12. A random marked point process $\left(\mathcal{E}^{*}, \mathfrak{X}^{*}, P^{*}\right)$ is called simple in a bounded space

$$
\left(Y, \mathfrak{A}_{Y}, \mathfrak{B}_{Y}\right)
$$

if almost all its trajectories $E^{*}=\left(\left[x_{1} ; k_{1}\right], \ldots,\left[x_{n} ; k_{n}\right], \ldots\right)$ are such that $x_{i} \neq x_{j}, i \neq j$, that is

for all $x \in X$, where

$$
P^{*}\left\{E^{*}: N^{*}\left(E^{*},\{x\} \times K\right) \leq 1\right\}=1
$$

$$
N^{*}\left(E^{*}, B_{Y}\right)=\operatorname{card}\left[E^{*} \cap B_{Y}\right]
$$

is a random counting measure of the marked point process $\left(B_{Y} \in \mathfrak{E}_{y}=\mathfrak{A}_{Y} \cap \mathfrak{B}_{Y}\right)$.

Definition 2.13. A random marked point process $\left(\mathcal{E}^{*}, \mathfrak{X}^{*}, P^{*}\right)$ is called strictly simple in a bounded space $\left(Y, \mathfrak{A}_{Y}, \mathfrak{B}_{Y}\right)$ if $x_{i} \neq x_{j}$ and $k_{i} \neq k_{j}, i \neq j$, for almost all its trajectories $E^{*}$, that is

$$
\begin{gathered}
P^{*}\left\{E^{*}: N^{*}\left(E^{*},\{x\} \times K\right) \leq 1\right\}=1, \\
P^{*}\left\{E^{*}: N^{*}\left(E^{*}, X \times\{k\}\right) \leq 1\right\}=1
\end{gathered}
$$

for all $x \in X$ and $k \in K$.

Definition 2.14. A random marked point process $\left(\mathcal{E}^{*}, \mathfrak{X}^{*}, P^{*}\right)$ is called finite in a bounded space

$$
\left(Y, \mathfrak{A}_{Y}, \mathfrak{B}_{Y}\right)
$$

if the size of almost all its trajectories $E^{*}$ is finite with probability one,

$$
P^{*}\left\{E^{*}: \operatorname{card}\left[E^{*}\right]=N^{*}\left(E^{*}, Y\right)<\infty\right\}=1 .
$$


Definition 2.15. $\left(\mathcal{E}^{*}, \mathfrak{X}^{*}, P^{*}\right)$ is called a simple finite ordered marked point process (ordered marked point process) in a bounded space

$$
\left(Y=X \times K, \mathfrak{A}_{Y}=\mathfrak{A}_{X} \otimes \mathfrak{A}_{K}, \mathfrak{B}_{Y}=\mathfrak{B}_{X} \odot \mathfrak{B}_{K}\right)
$$

if its projection $(\mathcal{E}, \mathfrak{X}, P)=P_{r_{X}}\left(\mathcal{E}^{*}, \mathfrak{X}^{*}, P^{*}\right)$ onto the space of positions $X$ is a finite simple ordered point process in the bounded space of positions $\left(X, \mathfrak{A}_{X}, \mathfrak{B}_{X}\right)[19$.

An arbitrary trajectory $E^{*}$ of an ordered marked point process $\left(\mathcal{E}^{*}, \mathfrak{X}^{*}, P^{*}\right)$ is a finite simple ordered thinned set (vector) of the phase space $Y=X \times K$,

$$
E^{*}=\left(y_{1}, \ldots, y_{i}, \ldots, y_{n}\right)=\left(\left[x_{1} ; k_{1}\right], \ldots,\left[x_{i} ; k_{i}\right], \ldots,\left[x_{n} ; k_{n}\right]\right),
$$

where $x_{i}$ is a position and where $k_{i}$ is the mark of the random event $y_{i}=\left[x_{i} ; k_{i}\right]$.

\section{MAIN DEFINITIONS OF THE THEORY OF MIXED EMPIRICAL ORDERED POINT PROCESSES}

In this section, we recall basic notions of the theory of mixed empirical ordered point processes in compact metric spaces [10, 11, 20, 21.

Assume that $\left(X, \mathfrak{A}_{X}, \vartheta\right)$ is a measurable compact metric space $\left(X, \mathfrak{A}_{X}\right)$ with a measure $\vartheta$, metric $\rho_{X}\left(x_{i}, x_{j}\right)$, and natural structures of measurable sets $\mathfrak{A}_{X}$ and bounded sets $\mathfrak{B}_{X}$ [10. In particular, $\vartheta$ can be a Lebesgue measure or its analogue if the metric space $X$ is a compact oriented differentiable manifold (sphere, ellipsoid, etc.). See [22, 23].

Every trajectory $E$ of a finite simple ordered point process $\widetilde{\mathcal{D}}=(\mathfrak{E}, \mathfrak{X}, \mathrm{P})$ in a bounded space $\left(X, \mathfrak{A}_{X}, \mathfrak{B}_{X}\right)$ is a thinned set of the metric space $X$ constituted by finite sequences of points: $E=\left(x_{1}, \ldots, x_{i}, \ldots, x_{n}\right)$, where $x_{i}$ is a position $\left(x_{i} \in X, i=1, \ldots, n\right)$.

Consider the following method of constructing an ordered point process. Introduce two random variables $x=x(\omega)$ and $\nu=\nu(\omega)$ such that

$3.1)$ the random variable $x(\omega)$ and integer valued nonnegative random variable $\nu(\omega)$ are defined in a certain main probability space $(\Omega=\{\omega\}, \Im, P)$;

$3.2)$ the random variables $x(\omega)$ and $\nu(\omega)$ assume values in sample spaces $\left(X, \mathfrak{A}_{X}, P_{x}\right)$ and $\left(Z_{+}, \mathfrak{A}_{Z_{+}}, P_{\nu}\right)$, respectively, endowed with probability measures $P_{x}$ and $P_{\nu}$, where

$$
\begin{gathered}
P_{x}\left(B_{X}\right)=\mathrm{P}\left\{\omega: x(\omega) \in B_{X}\right\}=\mu_{1}\left(B_{X}\right)\left(B_{X} \in \mathfrak{A}_{X}\right), \\
P_{\nu}\left(B_{Z_{+}}\right)=\mathrm{P}\left\{\omega: \nu(\omega) \in B_{Z_{+}}\right\}\left(B_{Z_{+}} \in \mathfrak{A}_{Z_{+}}\right) ;
\end{gathered}
$$

3.3) the distribution $P_{x}\left(B_{X}\right)$ of the random variable $x=x(\omega)$ is absolutely continuous with respect to the measure $\vartheta$ in the measurable space $\left(X, \mathfrak{A}_{X}\right)$;

3.4) $x(\omega)$ and $\nu(\omega)$ are independent random variables.

A number $n \in Z_{+}$is chosen randomly according to the distribution $P_{\nu}$ of the random variable $\nu(\omega)$. Then every trajectory $E=\left(x_{1}, \ldots, x_{i}, \ldots, x_{n}\right)$ of size $n$ of a simple ordered point process $(\mathcal{E}, \mathfrak{X}, P)$ is obtained by $n$ independent repetitions of the same random experiment $G_{1}$ being the random choice without repetition of a random point $x$ of the space $X$. The random experiment $G_{1}$ corresponds to the probability space $\left(X, \mathfrak{A}_{X}, P_{x}\right)$.

Hence the trajectory $E$ can be viewed as a realization of a finite simple ordered thinned random set (vector)

$$
E=E(\omega)=\left(x_{1}(\omega), \ldots, x_{i}(\omega), \ldots, x_{\nu(\omega)}(\omega)\right)
$$


in the measurable space $\left(X, \mathfrak{A}_{X}\right)$. The size $\nu(\omega)$ of the sample is random and the sample consists of independent identically distributed random variables defined in the probability space $(\Omega, \mathfrak{F}, \mathrm{P})$ with the distribution $P_{x}\left[\begin{array}{l}10 \\ {[10}\end{array}\right.$. It is easy to see that the random variable $\nu(\omega)$ admits the following representation

$$
\nu(\omega)=N=N(E, X)=\operatorname{card}[E \cap X]=\sum_{x \in E} I_{X}(x),
$$

where $N(E, X)$ is the random variable determining the number of points of the set $E$ in the space $X$ and where $I_{X}(\cdot)$ is the characteristic function of the space $X$.

Definition 3.1. A random process $\widetilde{\mathcal{D}}=(\mathcal{E}, \mathfrak{X}, \mathrm{P})$ is called a simple mixed empirical ordered point process of positions in a bounded space $\left(X, \mathfrak{A}_{X}, \mathfrak{B}_{X}\right)$.

Given a sample of size $n$ (that is, $N=n$ ) and arbitrary bounded measurable sets $B_{X}^{1} \in \mathfrak{E}_{X}$ and $B_{X}^{2}=X \backslash B_{X}^{1} \in \mathfrak{E}_{X}\left(\mathfrak{E}_{X}=\mathfrak{A}_{X} \cap \mathfrak{B}_{X}\right)$, the random empirical counting measure is defined by

$$
N\left(B_{X}^{1}\right)=N\left(E, B_{X}^{1}\right)=\operatorname{card}\left[E \cap B_{X}^{1}\right]=\sum_{i=1}^{n} I_{B_{X}^{1}}\left(x_{i}\right) .
$$

The conditional distribution of the counting measure $N\left(B_{X}^{1}\right)$ is binomial $B\left(n, \mu_{1}\left(B_{X}^{1}\right)\right)$ with the parametric measure $\mu_{1}\left(B_{X}^{1}\right)$ [10, 20]:

$$
\begin{aligned}
\mathrm{P}\left\{N\left(B_{X}^{1}\right)=k \mid N=n\right\} & =\mathrm{P}\left\{N\left(B_{X}^{1}\right)=k, N\left(B_{X}^{2}\right)=n-k \mid N=n\right\} \\
& =C_{n}^{k} \mu_{1}^{k}\left(B_{X}^{1}\right)\left[1-\mu_{1}\left(B_{X}^{1}\right)\right]^{n-k} \\
& =C_{n}^{k} \mu_{1}^{k}\left(B_{X}^{1}\right) \mu_{1}^{n-k}\left(B_{X}^{2}\right),
\end{aligned}
$$

where $\mu_{1}\left(B_{X}^{2}\right)=1-\mu_{1}\left(B_{X}^{1}\right), k=0,1, \ldots, n$. If $B_{X}^{1}, \ldots, B_{X}^{s}, \bigcup_{j=1}^{s} B_{X}^{j}=X$, is an arbitrary finite sequence of bounded measurable disjoint sets of the space $X, k_{j} \in Z_{+}$, $j=1, \ldots, s, \sum_{j=1}^{s} k_{j}=n$, then, given $n$ (that is, $N=n$ ), the conditional joint distribution of counting measures $N\left(B_{X}^{j}\right), j=1, \ldots, s$, is polynomial [21]:

$$
\mathrm{P}\left\{N\left(B_{X}^{j}\right)=k_{j}, j=1, \ldots, s \mid N=n\right\}=\frac{n !}{k_{1} ! \ldots k_{s} !} \mu_{1}^{k_{1}}\left(B_{X}^{1}\right) \ldots \mu_{1}^{k_{s}}\left(B_{X}^{s}\right),
$$

where

$$
\sum_{j=1}^{s} \mu_{1}\left(B_{X}^{j}\right)=1
$$

Using conditional distributions (2) and (3) and the full probability formula, we are able to evaluate the distribution of the counting measure $N\left(B_{X}^{1}\right)$ and joint distribution of counting measures

Indeed,

$$
\left\{N\left(B_{X}^{j}\right), j=1, \ldots, s\right\}
$$

$$
\begin{aligned}
\mathrm{P}\left\{N\left(B_{X}^{1}\right)=k\right\} & =\sum_{n \geq k} \mathrm{P}\left\{N\left(B_{X}^{1}\right)=k \mid N=n\right\} \mathrm{P}\{N=n\} \\
& =\sum_{n \geq k} C_{n}^{k} \mu_{1}^{k}\left(B_{X}^{1}\right) \mu_{1}^{n-k}\left(B_{X}^{2}\right) \mathrm{P}\{N=n\}, \quad k \in Z_{+},
\end{aligned}
$$

\footnotetext{
${ }^{1}$ We suppose that the metric space $X$ is a continuum with a continuous measure $P_{x}$; thus if an element is dropped from $X$, then the independence of members of the sample as well as their marginal distribution does not change.
} 


$$
\begin{aligned}
\mathrm{P}\{ & \left.N\left(B_{X}^{j}\right)=k_{j}, j=1, \ldots, s\right\} \\
& =\sum_{n=0}^{\infty} \mathrm{P}\left\{N\left(B_{X}^{j}\right)=k_{j}, j=1, \ldots, s \mid N=n\right\} \mathrm{P}\{N=n\} \\
& =\sum_{n=0}^{\infty} \frac{n !}{k_{1} ! \ldots k_{s} !} \mu_{1}^{k_{1}}\left(B_{X}^{1}\right) \ldots \mu_{1}^{k_{s}}\left(B_{X}^{s}\right) \mathrm{P}\{N=n\}, \quad \sum_{j=1}^{n} k_{j}=n, k_{j} \in Z_{+} .
\end{aligned}
$$

\section{MOMENT MEASURES OF MIXED EMPIRICAL RANDOM ORDERED POINT PROCESSES}

Now we establish the covariance measure describing the dependence between random counting measures $N\left(B_{X}^{1}\right)$ and $N\left(B_{X}^{2}\right)$ of an ordered point process $\widetilde{\mathcal{D}}=(\mathcal{E}, \mathfrak{X}, \mathrm{P})$ for two subsets $B_{X}^{1}$ and $B_{X}^{2}, B_{X}^{2}=X \backslash B_{X}^{1} \in \mathfrak{E}_{X}$, of the bounded space $\left(X, \mathfrak{A}_{X}, \mathcal{B}_{X}\right)$ :

(6) $\operatorname{cov}\left[N\left(B_{X}^{1}\right), N\left(B_{X}^{2}\right)\right]=\mathrm{E}\left[N\left(B_{X}^{1}\right) N\left(B_{X}^{2}\right)\right]-\mathrm{E}\left[N\left(B_{X}^{1}\right)\right] \mathrm{E}\left[N\left(B_{X}^{2}\right)\right]$.

First we evaluate the moment $\mathrm{E}\left[N\left(B_{X}^{1}\right) N\left(B_{X}^{2}\right)\right]$ by using the so-called full expectation formula 24]:

$$
\begin{aligned}
\mathrm{E}\left[N\left(B_{X}^{1}\right) N\left(B_{X}^{2}\right)\right] & =\mathrm{E}\left\{\mathrm{E}\left[N\left(B_{X}^{1}\right) N\left(B_{X}^{2}\right) \mid N=n\right]\right\} \\
& =\sum_{n=0}^{\infty} \mathrm{E}\left[N\left(B_{X}^{1}\right) N\left(B_{X}^{2}\right) \mid N=n\right] \mathrm{P}\{N=n\} .
\end{aligned}
$$

Considering (2) we obtain

$$
\begin{aligned}
\mathrm{E}[N & \left.\left(B_{X}^{1}\right) N\left(B_{X}^{2}\right) \mid N=n\right] \\
& =\sum_{k=0}^{n} k(n-k) \mathrm{P}\left\{N\left(B_{X}^{1}\right) N\left(B_{X}^{2}\right)=k(n-k) \mid N=n\right\} \\
& =\sum_{k=0}^{n} k(n-k) \mathrm{P}\left\{N\left(B_{X}^{1}\right)=k, N\left(B_{X}^{2}\right)=n-k \mid N=n\right\} \\
& =\sum_{k=0}^{n} k(n-k) \frac{n !}{k !(n-k) !} \mu_{1}^{k}\left(B_{X}^{1}\right) \mu_{1}^{n-k}\left(B_{X}^{2}\right) \\
& =n(n-1) \mu_{1}\left(B_{X}^{1}\right) \mu_{1}\left(B_{X}^{2}\right) \sum_{k=1}^{n-1} \frac{(n-2) !}{(k-1) !(n-k-1) !} \mu_{1}^{k-1}\left(B_{X}^{1}\right) \mu_{1}^{n-k-1}\left(B_{X}^{2}\right) \\
& =n(n-1) \mu_{1}\left(B_{X}^{1}\right) \mu_{1}\left(B_{X}^{2}\right)\left[\mu_{1}\left(B_{X}^{1}\right)+\mu_{1}\left(B_{X}^{2}\right)\right]^{n-2} \\
& =n(n-1) \mu_{1}\left(B_{X}^{1}\right) \mu_{1}\left(B_{X}^{2}\right)
\end{aligned}
$$

since $\mu_{1}\left(B_{X}^{1}\right)+\mu_{1}\left(B_{X}^{2}\right)=1$. Substituting equality (8) in formulas (6) and (7) we are able to evaluate the covariance:

$$
\operatorname{cov}\left[N\left(B_{X}^{1}\right), N\left(B_{X}^{2}\right)\right]=\mu_{1}\left(B_{X}^{1}\right) \mu_{1}\left(B_{X}^{2}\right) m_{[2]}-\mathrm{E}\left[N\left(B_{X}^{1}\right)\right] \mathrm{E}\left[N\left(B_{X}^{2}\right)\right],
$$

where

$$
m_{[2]}=\sum_{n=0}^{\infty} n(n-1) \mathrm{P}\{N=n\}=\mathrm{E}[N(N-1)]=\mathrm{E}\left[N^{[2]}\right]
$$

is the second factorial moment of the random variable $N$ and where $N^{[2]}=N(N-1)$ is the factorial power of order two [10, 24].

Next we construct the joint probability generating function

$$
\Pi_{N\left(B_{X}^{1}\right), \ldots, N\left(B_{X}^{s}\right)}\left(z_{1}, \ldots, z_{s}\right)=\mathrm{E}\left[z_{1}^{N\left(B_{X}^{1}\right)} \ldots z_{s}^{N\left(B_{X}^{s}\right)}\right]
$$


of random empirical counting measures $\left\{N\left(B_{X}^{j}\right), j=1, \ldots, s\right\}$ if $\bigcup_{j=1}^{s} B_{X}^{j}=X$ and $B_{X}^{i} \cap B_{X}^{r}=\varnothing, 1 \leq i, r \leq s, i \neq r$. We use the conditional joint distribution (3):

$$
\begin{aligned}
& \Pi_{N\left(B_{X}^{1}\right), \ldots, N\left(B_{X}^{s}\right)}\left(z_{1}, \ldots, z_{s}\right)=\mathrm{E}\left\{\mathrm{E}\left[z_{1}^{N\left(B_{X}^{1}\right)} \ldots z_{s}^{N\left(B_{X}^{s}\right)} \mid N=n\right]\right\} \\
& =\sum_{n=0}^{\infty} \mathrm{E}\left[z_{1}^{N\left(B_{X}^{1}\right)} \ldots z_{s}^{N\left(B_{X}^{s}\right)} \mid N=n\right] \mathrm{P}\{N=n\} \\
& =\sum_{n=0}^{\infty}\left\{\sum_{k_{1}, \ldots, k_{s} \geq 0, k_{1}+\cdots+k_{s}=n} z_{1}^{k_{1}} \ldots z_{s}^{k_{s}}\right. \\
& \left.\times \mathrm{P}\left\{N\left(B_{X}^{j}\right)=k_{j}, j=1, \ldots, s \mid N=n\right\}\right\} \mathrm{P}\{N=n\} \\
& =\sum_{n=0}^{\infty}\left\{\sum_{k_{1}, \ldots, k_{s} \geq 0, k_{1}+\cdots+k_{s}=n} \frac{n !}{k_{1} ! \ldots k_{s} !}\left[z_{1} \mu_{1}\left(B_{X}^{1}\right)\right]^{k_{1}} \ldots\left[z_{s} \mu_{1}\left(B_{X}^{s}\right)\right]^{k_{s}}\right\} \\
& \times \mathrm{P}\{N=n\} \\
& =\sum_{n=0}^{\infty}\left\{\sum_{j=1}^{s} z_{j} \mu_{1}\left(B_{X}^{j}\right)\right\}^{n} \mathrm{P}\{N=n\}=\mathrm{E}\left[\left\{\sum_{j=1}^{s} z_{j} \mu_{1}\left(B_{X}^{j}\right)\right\}^{N}\right] \\
& =\Pi_{N}\left(z_{1} \mu_{1}\left(B_{X}^{1}\right)+\cdots+z_{s} \mu_{1}\left(B_{X}^{s}\right)\right),
\end{aligned}
$$

where $\Pi_{N}(z)=\mathrm{E}\left[z^{N}\right]$ is the probability generating function of the random variable $N$.

The joint probability generating function (10) allows us to evaluate the marginal probability generating functions of the counting measures:

$$
\begin{aligned}
& \Pi_{N\left(B_{X}^{j}\right)}\left(z_{j}\right)=\mathrm{E}\left[z_{j}^{\left.N\left(B_{X}^{j}\right)\right]}=\Pi_{N}\left(z_{j} \mu_{1}\left(B_{X}^{j}\right)+\sum_{t=1, t \neq j}^{s} \mu_{1}\left(B_{X}^{t}\right)\right), \quad j=1, \ldots, s\right. \\
& \Pi_{N\left(B_{X}^{j_{1}}\right), \ldots, N\left(B_{X}^{j_{h}}\right)}\left(z_{j_{1}}, \ldots, z_{j_{h}}\right)=\mathrm{E}\left[z_{j_{1}}^{N\left(B_{X}^{j_{1}}\right)} \ldots z_{j_{h}}^{N\left(B_{X}^{j_{h}}\right)}\right] \\
&=\Pi_{N}\left(\sum_{t=1}^{h} z_{j_{t}} \mu_{1}\left(B_{X}^{j_{t}}\right)+\sum_{t=1, t \neq j_{1}, \ldots, j_{h}}^{s} \mu_{1}\left(B_{X}^{t}\right)\right)
\end{aligned}
$$

where $1 \leq j_{1}<\cdots<j_{h} \leq s, h=1, \ldots, s$, and their derivatives

$$
\begin{gathered}
\Pi_{N\left(B_{X}^{j}\right)}^{(h)}(1)=\mu_{1}^{h}\left(B_{X}^{j}\right) \Pi_{N}^{(h)}(1), \\
\left.\frac{\partial^{h}\left\{\Pi_{N\left(B_{X}^{j_{1}}\right), \ldots, N\left(B_{X}^{j_{h}}\right)}\left(z_{j_{1}}, \ldots, z_{j_{h}}\right)\right\}}{\partial z_{j_{1}} \ldots \partial z_{j_{h}}}\right|_{z_{j_{k}}=1, k=1, \ldots, h} \\
=\mu_{1}\left(B_{X}^{j_{1}}\right) \ldots \mu_{1}\left(B_{X}^{j_{h}}\right) \Pi_{N}^{(h)}(1),
\end{gathered}
$$

where $\Pi_{N}^{(h)}(\cdot)$ denotes the derivative of order $h$ of the probability generating function $\Pi_{N}(\cdot)$.

Below is the list of moment measures of an ordered point process $\widetilde{\mathcal{D}}$ :

(a): $\nu_{\widetilde{\mathcal{D}}}^{(h)}\left(B_{X}^{j}\right)=\mathrm{E}\left[N^{h}\left(B_{X}^{j}\right)\right]$ denotes the moment measure of order $h, h=1,2, \ldots$, 
(b): $\nu_{\widetilde{\mathcal{D}}}^{(h)}\left(B_{X}^{j_{1}} \times \cdots \times B_{X}^{j_{h}}\right)=\mathrm{E}\left[N\left(B_{X}^{j_{1}}\right) \ldots N\left(B_{X}^{j_{h}}\right)\right]$ is the mixed moment measure of order $h$,

(c): $\alpha_{\widetilde{\mathcal{D}}}^{(h)}\left(B_{X}^{j}\right)=\mathrm{E}\left[N^{[h]}\left(B_{X}^{j}\right)\right]=\mathrm{E}\left[N\left(B_{X}^{j}\right)\left(N\left(B_{X}^{j}\right)-1\right) \ldots\left(N\left(B_{X}^{j}\right)-h+1\right)\right]$ is the factorial moment measure of order $h$,

(d): $\nu_{\widetilde{\mathcal{D}}}^{(2)}\left(B_{X}^{j_{1}} \times B_{X}^{j_{2}}\right)=\mathrm{E}\left[N\left(B_{X}^{j_{1}}\right) N\left(B_{X}^{j_{2}}\right)\right]$ is the mixed moment measure of the second order, $1 \leq j_{1}, j_{2} \leq s, j_{1} \neq j_{2}$,

(e): $D\left(N\left(B_{X}^{j}\right)\right)=\nu_{\widetilde{\mathcal{D}}}^{(2)}\left(B_{X}^{j}\right)-\left[\nu_{\widetilde{\mathcal{D}}}^{(1)}\left(B_{X}^{j}\right)\right]^{2}$ is the variance of the counting measure $N\left(B_{X}^{j}\right)$,

(f): $\operatorname{cov}\left[N\left(B_{X}^{j_{1}}\right), N\left(B_{X}^{j_{2}}\right)\right]=\nu_{\widetilde{\mathcal{D}}}^{(2)}\left(B_{X}^{j_{1}} \times B_{X}^{j_{2}}\right)-\nu_{\widetilde{\mathcal{D}}}^{(1)}\left(B_{X}^{j_{1}}\right) \nu_{\widetilde{\mathcal{D}}}^{(1)}\left(B_{X}^{j_{2}}\right)$ is the covariance measure describing the dependence between the counting measures $N\left(B_{X}^{j_{1}}\right)$ and $N\left(B_{X}^{j_{2}}\right), 1 \leq j_{1}, j_{2} \leq s ; j_{1} \neq j_{2}$.

Since

$$
\begin{gathered}
\nu_{\widetilde{\mathcal{D}}}^{(h)}\left(B_{X}^{j_{1}} \times \cdots \times B_{X}^{j_{h}}\right)=\left.\frac{\partial^{h}\left\{\Pi_{N\left(B_{X}^{j_{1}}\right), \ldots, N\left(B_{X}^{j_{h}}\right)}\left(z_{j_{1}}, \ldots, z_{j_{h}}\right)\right\}}{\partial z_{j_{1}} \ldots \partial z_{j_{h}}}\right|_{z_{j_{1}}=1, \ldots, z_{j_{h}}=1}, \\
\alpha_{\widetilde{\mathcal{D}}}^{(h)}\left(B_{X}^{j}\right)=\Pi_{N\left(B_{X}^{j}\right)}^{(h)}(1), \nu_{\widetilde{\mathcal{D}}}^{(1)}\left(B_{X}^{j}\right)=\mathrm{E}\left[N\left(B_{X}^{j}\right)\right]=\Pi_{N\left(B_{X}^{j}\right)}^{\prime}(1), \\
\nu_{\widetilde{\mathcal{D}}}^{(2)}\left(B_{X}^{j}\right)=\mathrm{E}\left[N^{2}\left(B_{X}^{j}\right)\right]=\Pi_{N\left(B_{X}^{j}\right)}^{\prime \prime}(1)+\Pi_{N\left(B_{X}^{j}\right)}^{\prime}(1), \\
D\left(N\left(B_{X}^{j}\right)\right)=\Pi_{N\left(B_{X}^{j}\right)}^{\prime \prime}(1)-\left\{\Pi_{N\left(B_{X}^{j}\right)}^{\prime}(1)\right\}^{2}+\Pi_{N\left(B_{X}^{j}\right)}^{\prime}(1), \\
\nu_{\widetilde{\mathcal{D}}}^{(2)}\left(B_{X}^{j_{1}} \times B_{X}^{j_{2}}\right)=\left.\frac{\partial^{2}\left\{\Pi_{N\left(B_{X}^{j_{1}}\right), N\left(B_{X}^{j_{2}}\right)}\left(z_{j_{1}}, z_{j_{2}}\right)\right\}}{\partial z_{j_{1}} \partial z_{j_{2}}}\right|_{z_{j_{1}}=1, z_{j_{2}}=1},
\end{gathered}
$$

we use the derivatives given by (11) and (12) and evaluate the moment characteristic of the ordered point process $\widetilde{\mathcal{D}}=(\mathcal{E}, \mathfrak{X}, \mathrm{P})$ of positions:

$$
\begin{gathered}
\nu_{\widetilde{\mathcal{D}}}^{(h)}\left(B_{X}^{j_{1}} \times \cdots \times B_{X}^{j_{h}}\right)=\mu_{1}\left(B_{X}^{j_{1}}\right) \ldots \mu_{1}\left(B_{X}^{j_{h}}\right) \Pi_{N}^{(h)}(1) \\
\alpha_{\widetilde{\mathcal{D}}}^{(h)}\left(B_{X}^{j}\right)=\mu_{1}^{h}\left(B_{X}^{j}\right) \Pi_{N}^{(h)}(1), \nu_{\widetilde{\mathcal{D}}}^{(1)}\left(B_{X}^{j}\right)=\mu_{1}\left(B_{X}^{j}\right) \Pi_{N}^{\prime}(1) \\
\nu_{\widetilde{\mathcal{D}}}^{(2)}\left(B_{X}^{j}\right)=\mu_{1}^{2}\left(B_{X}^{j}\right) \Pi_{N}^{\prime \prime}(1)+\mu_{1}\left(B_{X}^{j}\right) \Pi_{N}^{\prime}(1) \\
\nu_{\widetilde{\mathcal{D}}}^{(2)}\left(B_{X}^{j_{1}} \times B_{X}^{j_{2}}\right)=\mu_{1}\left(B_{X}^{j_{1}}\right) \mu_{1}\left(B_{X}^{j_{2}}\right) \Pi_{N}^{\prime \prime}(1) \\
D\left(N\left(B_{X}^{j}\right)\right)=\mu_{1}^{2}\left(B_{X}^{j}\right)\left[\Pi_{N}^{\prime \prime}(1)-\left\{\Pi_{N}^{\prime}(1)\right\}^{2}\right]+\mu_{1}\left(B_{X}^{j}\right) \Pi_{N}^{\prime}(1) \\
\operatorname{cov}\left[N\left(B_{X}^{j_{1}}\right), N\left(B_{X}^{j_{2}}\right)\right]=\mu_{1}\left(B_{X}^{j_{1}}\right) \mu_{1}\left(B_{X}^{j_{2}}\right)\left[\Pi_{N}^{\prime \prime}(1)-\left\{\Pi_{N}^{\prime}(1)\right\}^{2}\right]
\end{gathered}
$$

Remark 4.1. If $s=2$, then formula (10) allows us to establish the joint probability generating function of the counting measures $N\left(B_{X}^{1}\right)$ and $N\left(B_{X}^{2}\right)$,

$$
\Pi_{N\left(B_{X}^{1}\right), N\left(B_{X}^{2}\right)}\left(z_{1}, z_{2}\right)=\Pi_{N}\left(z_{1} \mu_{1}\left(B_{X}^{1}\right)+z_{2} \mu_{1}\left(B_{X}^{2}\right)\right),
$$

and the marginal probability generating function of the random variables $N\left(B_{X}^{1}\right)$ and $N\left(B_{X}^{2}\right)$ :

$$
\begin{aligned}
& \Pi_{N\left(B_{X}^{1}\right)}\left(z_{1}\right)=\Pi_{N\left(B_{X}^{1}\right), N\left(B_{X}^{2}\right)}\left(z_{1}, 1\right)=\Pi_{N}\left(1+\mu_{1}\left(B_{X}^{1}\right)\left(z_{1}-1\right)\right), \\
& \Pi_{N\left(B_{X}^{2}\right)}\left(z_{2}\right)=\Pi_{N\left(B_{X}^{1}\right), N\left(B_{X}^{2}\right)}\left(1, z_{2}\right)=\Pi_{N}\left(1+\mu_{1}\left(B_{X}^{2}\right)\left(z_{2}-1\right)\right) .
\end{aligned}
$$


See 25. Since

$$
m_{[2]}=\mathrm{E}\left[N^{[2]}\right]=\Pi_{N}^{\prime \prime}(1), \quad \mathrm{E}\left[N\left(B_{X}^{1}\right)\right]=\Pi_{N\left(B_{X}^{1}\right)}^{\prime}(1)=\mu_{1}\left(B_{X}^{1}\right) \Pi_{N}^{\prime}(1),
$$

and $\mathrm{E}\left[N\left(B_{X}^{2}\right)\right]=\Pi_{N\left(B_{X}^{2}\right)}^{\prime}(1)=\mu_{1}\left(B_{X}^{2}\right) \Pi_{N}^{\prime}(1)$, equality (9) implies that

$$
\operatorname{cov}\left[N\left(B_{X}^{1}\right), N\left(B_{X}^{2}\right)\right]=\mu_{1}\left(B_{X}^{1}\right) \mu_{1}\left(B_{X}^{2}\right)\left[\Pi_{N}^{\prime \prime}(1)-\left\{\Pi_{N}^{\prime}(1)\right\}^{2}\right] .
$$

Remark 4.2 (21]). If a finite sequence of bounded measurable disjoint sets $B_{X}^{1}, \ldots, B_{X}^{s}$ in the space $X$ is not a partition of the space $X$, that is, if $\bigcup_{j=1}^{s} B_{X}^{j} \neq X$, then the joint probability generating function is given by

$$
\Pi_{N\left(B_{X}^{1}\right), \ldots, N\left(B_{X}^{s}\right)}\left(z_{1}, \ldots, z_{s}\right)=\Pi_{N}\left(\sum_{j=1}^{s}\left(z_{j}-1\right) \mu_{1}\left(B_{X}^{j}\right)+1\right) .
$$

If the distribution of the random variable $N$ is Poissonian with parameter $\lambda$, then the joint probability generating function (20) is written as follows

$$
\Pi_{N\left(B_{X}^{1}\right), \ldots, N\left(B_{X}^{s}\right)}\left(z_{1}, \ldots, z_{s}\right)=\exp \left\{\sum_{j=1}^{s} \lambda \mu_{1}\left(B_{X}^{j}\right)\left(z_{j}-1\right)\right\} .
$$

See 24 .

\section{Simple MiXed EMPIRICAL RANDOM Poisson ORDEREd POINT PROCESSES}

We construct the random empirical counting measure $N\left(B_{X}\right)$ of an ordered point process $\widetilde{\mathcal{D}}=(\mathcal{E}, \mathfrak{X}, \mathrm{P})$ :

$$
N\left(B_{X}\right)=N\left(E, B_{X}\right)=\operatorname{card}\left[E \cap B_{X}\right]=\sum_{i=1}^{\nu} I_{B_{X}}\left(x_{i}\right)\left(B_{X} \in \mathfrak{E}_{X}\right) .
$$

Theorem 5.1 ([20]). If

1. the size of a sample $\nu=\nu(\omega)=N$ of a simple mixed empirical ordered point process $\widetilde{\mathcal{D}}=(\mathcal{E}, \mathfrak{X}, \mathrm{P})$ is a random variable distributed according to the homogeneous Poisson law with parameter $\lambda$ :

$$
P_{\nu}\{\nu=k\}=\mathrm{P}\{N=k\}=\frac{\lambda^{k}}{k !} e^{-\lambda}, \quad k=0,1,2, \ldots ;
$$

2. the random variable $\nu$ is independent of the random variables

$$
\left\{x_{i}(\omega) ; i=1, \ldots, \nu(\omega)\right\} ;
$$

3. $\left\{B_{X}^{j}: j=1, \ldots, s, s \geq 2\right\}$ is an arbitrary finite sequence of bounded measurable disjoint subsets of the space $X$,

then

$1^{*}$. the empirical counting measure $N\left(B_{X}^{j}\right), j=1, \ldots, s$, has the Poisson distribution with the parametric measure $\lambda \mu_{1}\left(B_{X}^{j}\right)$, that is,

$$
\mathrm{P}\left\{N\left(B_{X}^{j}\right)=k_{j}\right\}=\frac{\left[\lambda \mu_{1}\left(B_{X}^{j}\right)\right]^{k_{j}}}{k_{j} !} e^{-\lambda \mu_{1}\left(B_{X}^{j}\right)},
$$

where $\mu_{1}\left(B_{X}^{j}\right)=P_{x}\left(B_{X}^{j}\right), k_{j}=0,1,2, \ldots$; 
$2^{*}$. the empirical counting measures $N\left(B_{X}^{1}\right), \ldots, N\left(B_{X}^{s}\right)$ are jointly independent random variables:

$$
\mathrm{P}\left\{N\left(B_{X}^{j}\right)=k_{j}, j=1, \ldots, s\right\}=\prod_{j=1}^{s} \mathrm{P}\left\{N\left(B_{X}^{j}\right)=k_{j}\right\} .
$$

Proof. We prove the first statement by using the full probability formula and the assumption that the distribution in (2) is binomial:

$$
\begin{aligned}
\mathrm{P}\left\{N\left(B_{X}^{j}\right)=k_{j}\right\} & =\sum_{n \geq k_{j}} \mathrm{P}\left\{N\left(B_{X}^{j}\right)=k_{j} \mid N=n\right\} \mathrm{P}\{N=n\} \\
& =\sum_{n \geq k_{j}} C_{n}^{k_{j}} \mu_{1}^{k_{j}}\left(B_{X}^{j}\right)\left(1-\mu_{1}\left(B_{X}^{j}\right)\right)^{n-k_{j}} \frac{\lambda^{n}}{n !} e^{-\lambda} \\
& =\sum_{\left(n-k_{j}\right) \geq 0} \frac{n !}{k_{j} !\left(n-k_{j}\right) !} \mu_{1}^{k_{j}}\left(B_{X}^{j}\right)\left(1-\mu_{1}\left(B_{X}^{j}\right)\right)^{n-k_{j}} \frac{\lambda^{n-k_{j}} \lambda^{k_{j}}}{n !} e^{-\lambda} \\
& =\frac{\left[\lambda \mu_{1}\left(B_{X}^{j}\right)\right]^{k_{j}}}{k_{j} !} e^{-\lambda} \sum_{m \geq 0} \frac{\left[\lambda\left(1-\mu_{1}\left(B_{X}^{j}\right)\right)\right]^{m}}{m !},
\end{aligned}
$$

$m=n-k_{j}$. Since

$$
\sum_{m \geq 0} \frac{\left[\lambda\left(1-\mu_{1}\left(B_{X}^{j}\right)\right)\right]^{m}}{m !}=e^{\lambda} e^{-\lambda \mu_{1}\left(B_{X}^{j}\right)}
$$

we get

$$
\mathrm{P}\left\{N\left(B_{X}^{j}\right)=k_{j}\right\}=\frac{\left[\lambda \mu_{1}\left(B_{X}^{j}\right)\right]^{k_{j}}}{k_{j} !} e^{-\lambda \mu_{1}\left(B_{X}^{j}\right)} .
$$

In this case, the probability generating function of the counting measure $N\left(B_{X}^{j}\right)$ can be written as follows

$$
\Pi_{N\left(B_{X}^{j}\right)}\left(z_{j}\right)=\exp \left\{\lambda \mu_{1}\left(B_{X}^{j}\right)\left(z_{j}-1\right)\right\}
$$

See 24 .

To prove the second statement of the theorem we use the joint probability generating function (21) of the Poisson counting measures $\left\{N\left(B_{X}^{j}\right), j=1, \ldots, s\right\}$ :

$$
\Pi_{N\left(B_{X}^{1}\right), \ldots, N\left(B_{X}^{s}\right)}\left(z_{1}, \ldots, z_{s}\right)=\exp \left\{\sum_{j=1}^{s} \lambda \mu_{1}\left(B_{X}^{j}\right)\left(z_{j}-1\right)\right\}=\prod_{j=1}^{s} \Pi_{N\left(B_{X}^{j}\right)}\left(z_{j}\right) .
$$

Since the joint probability generating function (23) is a product of probability generating functions (22) of the components, the random variables $N\left(B_{X}^{1}\right), \ldots, N\left(B_{X}^{s}\right)$ are jointly independent (see [25]).

Remark 5.1. Similar results can also be obtained for the case

$$
\bigcup_{j=1}^{s} B_{X}^{j}=X, \quad B_{X}^{i} \cap B_{X}^{r}=\varnothing, \quad 1 \leq i, r \leq s, i \neq r .
$$

Definition 5.1. A random process $\widetilde{\mathcal{D}}=(\mathcal{E}, \mathfrak{X}, P)$ is called a simple mixed empirical Poisson ordered point process of positions in a bounded space $\left(X, \mathfrak{A}_{X}, \mathcal{B}_{X}\right)$ if Theorem 5.1 holds. 
Corollary 5.1. Let the random variable $N$ have the Poisson distribution with parameter $\lambda$. Then its probability generating function is $\Pi_{N}(z)=\exp \{\lambda(z-1)\}$ and the derivatives of $\Pi_{N}(z)$ are given by

$$
\left\{\Pi_{N}^{(h)}(1)=\lambda^{h}: h=1,2, \ldots\right\} .
$$

Thus equalities (13)-(18) imply that

$$
\begin{gathered}
\nu_{\widetilde{\mathcal{D}}}^{(1)}\left(B_{X}^{j}\right)=\lambda \mu_{1}\left(B_{X}^{j}\right) \\
\nu_{\widetilde{\mathcal{D}}}^{(h)}\left(B_{X}^{j_{1}} \times \cdots \times B_{X}^{j_{h}}\right)=\lambda^{h} \mu_{1}\left(B_{X}^{j_{1}}\right) \ldots \mu_{1}\left(B_{X}^{j_{h}}\right)=\nu_{\widetilde{\mathcal{D}}}^{(1)}\left(B_{X}^{j_{1}}\right) \ldots \nu_{\widetilde{\mathcal{D}}}^{(1)}\left(B_{X}^{j_{h}}\right), \\
\alpha_{\widetilde{\mathcal{D}}}^{(h)}\left(B_{X}^{j}\right)=\lambda^{h} \mu_{1}^{h}\left(B_{X}^{j}\right)=\left[\nu_{\widetilde{\mathcal{D}}}^{(1)}\left(B_{X}^{j}\right)\right]^{h} \\
\nu_{\widetilde{\mathcal{D}}}^{(2)}\left(B_{X}^{j}\right)=\lambda^{2} \mu_{1}^{2}\left(B_{X}^{j}\right)+\lambda \mu_{1}\left(B_{X}^{j}\right) \\
\nu_{\widetilde{\mathcal{D}}}^{(2)}\left(B_{X}^{j_{1}} \times B_{X}^{j_{2}}\right)=\lambda^{2} \mu_{1}\left(B_{X}^{j_{1}}\right) \mu_{1}\left(B_{X}^{j_{2}}\right) \\
D\left(N\left(B_{X}^{j}\right)\right)=\lambda \mu_{1}\left(B_{X}^{j}\right), \quad \operatorname{cov}\left[N\left(B_{X}^{j_{1}}\right), N\left(B_{X}^{j_{2}}\right)\right]=0 .
\end{gathered}
$$

Remark 5.2. If the random variable $N$ has a compound Poisson distribution with the probability generating function

$$
\Pi_{N}(z)=\exp \{\lambda(\Pi(z)-1)\}, \quad \text { where } \Pi(z)=q+p z, \text { and } q=1-p
$$

is the probability generating function of a Bernoulli random variable with parameter $p$ [25], then the joint probability generating function of counting measures

$$
\left\{N\left(B_{X}^{j}\right), j=1, \ldots, s\right\}
$$

such that

$$
\bigcup_{j=1}^{s} B_{X}^{j}=X, \quad B_{X}^{i} \cap B_{X}^{r}=\varnothing, \quad 1 \leq i, r \leq s, i \neq r
$$

is given by

$$
\Pi_{N\left(B_{X}^{1}\right), \ldots, N\left(B_{X}^{s}\right)}\left(z_{1}, \ldots, z_{s}\right)=\exp \left\{\sum_{j=1}^{s} \lambda p \mu_{1}\left(B_{X}^{j}\right)\left(z_{j}-1\right)\right\} .
$$

\section{Mixed EMPiRicAl NEgAtive Binomial RANDOM ORDERED POINT PROCESSES}

Let the size $\nu=\nu(\omega)=N$ of a sample be a random variable with the negative binomial distribution with parameters $\alpha$ and $p$ [24, 26]:

$$
P_{\nu}\{\nu=k\}=\mathrm{P}\{N=k\}=C_{\alpha+k-1}^{k} p^{\alpha} q^{k}, \quad k \in Z_{+}, q=1-p .
$$

Then $\Pi_{N}(z)=p^{\alpha}(1-q z)^{-\alpha}$ is the probability generating function of $N$. Assume that $N$ is independent of all other random variables (11). Simple algebra reduces $\Pi_{N}(z)$ to a more convenient form

$$
\Pi_{N}(z)=\frac{p^{\alpha}}{[p+q(1-z)]^{\alpha}}=\frac{1}{[1+\beta(1-z)]^{\alpha}}, \quad \beta=\frac{q}{p}>0 .
$$

Definition 6.1. A random process $\widetilde{\mathcal{D}}=(\mathcal{E}, \mathfrak{X}, P)$ is called a simple mixed empirical negative binomial ordered point process in a bounded space $\left(X, \mathfrak{A}_{X}, B_{X}\right)$ if the size $N$ of a sample is a random variable with a negative binomial distribution. 
Using (10), (13)-(18), and (30) we get

$$
\begin{gathered}
\Pi_{N\left(B_{X}^{1}\right), \ldots, N\left(B_{X}^{s}\right)}\left(z_{1}, \ldots, z_{s}\right)=\left[1+\beta \sum_{j=1}^{s} \mu_{1}\left(B_{X}^{j}\right)\left(1-z_{j}\right)\right]^{-\alpha}, \\
\Pi_{N\left(B_{X}^{j}\right)}\left(z_{j}\right)=\left[1+\beta \mu_{1}\left(B_{X}^{j}\right)\left(1-z_{j}\right)\right]^{-\alpha}, \quad j=1, \ldots, s, \\
\nu_{\widetilde{\mathcal{D}}}^{(h)}\left(B_{X}^{j_{1}} \times \cdots \times B_{X}^{j_{h}}\right)=\beta^{h} \prod_{i=1}^{h}(\alpha+i-1) \mu_{1}\left(B_{X}^{j_{1}}\right) \ldots \mu_{1}\left(B_{X}^{j_{h}}\right), \\
\alpha_{\widetilde{\mathcal{D}}}^{(h)}\left(B_{X}^{j}\right)=\beta^{h} \prod_{i=1}^{h}(\alpha+i-1) \mu_{1}^{h}\left(B_{X}^{j}\right), \quad \nu_{\widetilde{\mathcal{D}}}^{(1)}\left(B_{X}^{j}\right)=\alpha \beta \mu_{1}\left(B_{X}^{j}\right), \\
\nu_{\widetilde{\mathcal{D}}}^{(2)}\left(B_{X}^{j}\right)=\alpha \beta \mu_{1}\left(B_{X}^{j}\right)\left[(\alpha+1) \beta \mu_{1}\left(B_{X}^{j}\right)+1\right], \\
\nu_{\widetilde{\mathcal{D}}}^{(2)}\left(B_{X}^{j_{1}} \times B_{X}^{j_{2}}\right)=\alpha(\alpha+1) \beta^{2} \mu_{1}\left(B_{X}^{j_{1}}\right) \mu_{1}\left(B_{X}^{j_{2}}\right), \\
D\left(N\left(B_{X}^{j}\right)\right)=\alpha \beta \mu_{1}\left(B_{X}^{j}\right)\left[\beta \mu_{1}\left(B_{X}^{j}\right)+1\right], \\
\operatorname{cov}\left[N\left(B_{X}^{j_{1}}\right), N\left(B_{X}^{j_{2}}\right)\right]=\alpha \beta^{2} \mu_{1}\left(B_{X}^{j_{1}}\right) \mu_{1}\left(B_{X}^{j_{2}}\right), \quad 1 \leq j_{1}, j_{2} \leq s, j_{1} \neq j_{2} .
\end{gathered}
$$

Looking at equalities (31)-(33) we conclude that

a) counting measures $N\left(B_{X}^{1}\right), \ldots, N\left(B_{X}^{s}\right)$ form a family of mutually correlated random variables whose distribution is negative binomial with parameters

$$
\beta \mu_{1}\left(B_{X}^{j}\right)>0, \quad \alpha>0, \quad j=1, \ldots, s ;
$$

b) there is a positive correlation between counting measures $N\left(B_{X}^{j_{1}}\right)$ and $N\left(B_{X}^{j_{2}}\right)$.

\section{BIBLIOGRAPHY}

1. N. G. Semeǐko, Yu. I. Petunin, and V. P. Yatsenko, Studying the morphometric characteristics of nuclear pore complexes of a sensory neuron using methods of spherical stochastic geometry, Kibernetika and Sistemnyi Analiz 42 (2006), no. 6, 175-182; English transl. in Cybernetics and Systems Analysis 42 (2006), no. 6, 917-922.

2. A. Baddeley and E. B. Vedel Jensen, Stereology for Statisticians, Chapman and Hall/CRC, New York, 2005. MR2107000 (2005g:62001)

3. G. S. Watson, Mathematical morfology, A Survey of Statistical Design and Linear Models (I. N. Srivastava, ed.), North-Holland Publishing Company, 1975, pp. 547-553. MR0378323 $(51: 14491)$

4. A. F. Karr, Point Processes and Their Statistical Inference, Marcel Dekker, New York, 1991. MR.1113698 (92f:62116)

5. M. Csörgő and P. Révész, Strong Approximation in Probability and Statistics, Academic Press, New York, 1981. MR666546 (84d:60050)

6. P. Gaenssler, Empirical Processes: On Some Basic Results from the Probabilistic Point of View, Institute of Mathematical Statistics, Hayward, CA, 1984.

7. P. Gaenssler and W. Stute, On uniform convergence of measures with application to uniform convergence of empirical distribution, Lect. Notes Math., vol. 566, 1976, pp. 45-56. MR0433534 (55:6510)

8. D. Pollard, Convergence of Stochastic Processes, Springer-Verlag, New York, 1984. MR762984 (86i:60074)

9. R. Serfling, Approximation Theorems of Mathematical Statistics, Wiley, New York, 1980. MR595165 (82a:62003)

10. Yu. I. Petunin and M. G. Semeı̌ko, Mixed empirical stochastic point processes in compact metric spaces. I, Teor. Imovir. Mat. Stat. 74 (2006), 98-107; English transl. in Theory Probab. Math. Statist. 74 (2007), 113-123. MR2321193(2008f:60053) 
11. Yu. I. Petunin and M. G. Semeı̌ko, Mixed empirical stochastic point processes in compact metric spaces. II, Teor. Imovir. Mat. Stat. 75 (2006), 121-126; English transl. in Theory Probab. Math. Statist. 74 (2007), 139-145. MR2321187(2008f:60054)

12. N. G. Semeǐko, Mixed empirical Poisson random spherical-cap process, Kibernetika ta sistemnyi analiz (2011), no. 5, 119-130; English transl. in Cybernetics and Systems Analysis 47 (2011), no. $5,773-782$.

13. J. E. Moyal, The general theory of stochastic population processes, Acta Math. 108 (1962), no. 1, 1-31. MR0148107 (26:5616)

14. B. D. Ripley, Locally finite random sets: foundations for point process theory, Ann. Probab. 4 (1976), no. 6, 983-994. MR0474478 (57:14117)

15. K. Matthes, J. Kerstan, and J. Mecke, Infinitely Divisible Point Processes, John Wiley \& Sons, Chichester-New York-Brisbane-Toronto 1978. MR0517931 (58:24538)

16. O. Kallenberg, Random Measures, Akademie-Verlag, Berlin, 1975. MR.0431372 (55:4372)

17. P. I. Daley, Various concepts of orderliness for point processes, Stochastic Geometry (R. F. Harding and P. G. Kendall, eds.), New York, 1974, pp. 148-164. MR0380976 (52:1873)

18. G. Birkhoff, Lattice Theory, American Mathematical Society Colloquium Publications, vol. 25, revised edition, American Mathematical Society, New York, 1948. MR0029876 (10:673a)

19. Yu. I. Petunin and N. G. Semeǐko, A random process of segments on a two-dimensional Euclidean sphere I, Teor. Veroyatnost. Mat. Statist. 39 (1988), 107-113; English transl. in Theory Probab. Math. Statist. 39 (1989), 129-135. MR947940 (89g:60170)

20. P. Gaenssler, Empirical Processes, Lecture Notes, Regional Monograph Series, vol. 2, Institute of Mathematical Statistics, Munich, 1983. MR744668 (86f:60044)

21. D. I. Daley and D. Vere-Jones, An Introduction to the Theory of Point Processes, SpringerVerlag, New York, 1988. MR950166 (90e:60060)

22. R L. Bishop and R. J. Crittenden, Geometry of Manifolds, Academic Press, New York, 1964. MR.0169148(29:6401)

23. Hassler Whitney, Geometric Integration Theory, Princeton University Press, Princeton, 1957. MR.0087148(19:309c)

24. V. S. Koroljuk, N. I. Portenko, A. V. Skorohod, and A. F. Turbin, A Manual on Probability Theory and Mathematical Statistics, "Naukova Dumka", Kiev, 1978. (Russian) MR502722 (80b:60002)

25. W. Feller, An Introduction to Probability Theory and Its Applications, vol. 1, Wiley, New York, 1950. MR0038583(12:424a)

26. N. A. J. Hastings and J. B. Peacock, Statistical Distributions: A Handbook for Students and Practitioners, Butterworth, London, 1975. MR0359137 (50:11592)

Department of Higher Mathematics, Faculty for Human Resources Management and Marketing, Kyiv National Vadym Get'man University for Economics, Peremogy Avenue, 54/1, KYIV 03680, UKRAINE

E-mail address: semejko@ukr.net 